\title{
DES EROTICA PATHÉMATA : VARIATIONS SUR LA PASSION D'AMOUR CHEZ PARTHÉNIOS DE NICÉE ET DANS LES GÉORGIQUES DE VIRGILE
}

\begin{abstract}
Summary: At the end of the Georgics Virgil represents himself as someone nursed by sweet Parthenope (IV 536: dulcis alebat / Parthenope). According to the rather obscure tradition which goes back to Servius, Parthenope would be an allusion to one of the Sirens, patron divinity of Naples-Parthenopolis, which was the favourite place of the poet. Nevertheless, Parthenope used to be considered as a self-referential joke on the nickname of Virgil, called Parthenias (a virgin) because of his moral excellence. The paper offers a new metapoetic reading of the passage which wishes to complete the earlier interpretations based on biographical data and local tradition. The allusion should also be regarded as a statement about inspiration. By suggesting a new approach to the mythology (see the Muse replaced by the Siren), the name of Parthenope appears to create an homage to Parthenius of Nicaea and to his strange collection of erotic myths. The studies about the impact of the Erotica pathemata on Latin poetry generally focus on the Elegiacs and Ovid. Nevertheless, it cannot be excluded that the mythological allusions of the Georgics about the origins of plants, animals, etc. may be influenced by some typical narrative patterns of Parthenius. The series of these virgilian aetological notes alluding to tragic love stories of Greek mythology seems to prepare the great Orpheus myth of Book IV. On the other hand, Virgil's short allusions might transmit a concept of human passion, which sometimes is rather similar to the emotional world of the Parthenian narratives, but which is always much more rich in ethical concerns.
\end{abstract}

Keywords: Virgil, Georgics, Parthenope, Parthenius of Nicaea, Erotica Pathemata, Servius, erotic myths

La sphragis des Géorgiques, le seul passage où Virgile se nomme ouvertement, met en relation le poète avec une certaine Parthénopé représentée comme une douce nourrice (IV 563-564) : Illo Vergilium me tempore dulcis alebat / Parthenope studiis florentem ignobilis oti. L'interprétation du passage n'est pas univoque. Selon une lecture contemporaine, l'allusion met en évidence la condition mortelle de l'homme et la passion humaine; selon une autre, elle évoquerait la douceur du paysage campanien si cher au poète. ${ }^{1}$ Conformément à Servius ${ }^{2}$ dont le commentaire est de première

\footnotetext{
${ }^{1}$ Voir MiLes, G. B. : Virgil's Georgics. A New Interpretation. Berkeley 1980, p. 293, cf. ERREN, M. : P. Vergilius Maro. Georgica. II. Heidelberg 2003, p. 1002.
} 
importance pour notre analyse, le nom de Parthénopé fait allusion à la ville de Naples, à l'ancienne Parthénopolis ${ }^{3}$ et à sa protectrice divine qui est généralement identifiée avec une des Sirènes. La tradition ancienne est partagée sur les circonstances exactes de l'arrivée de Parthénopé en Campanie. Selon la version née dans l'hellénisme, son culte napolitain remonte à la confrontation entre Ulysse et les trois filles-oiseaux au chant létal qui se suicident après l'échec de leur tentative d'empêcher le nostos du roi d'Ithaque. ${ }^{4}$ Transporté par les vagues, le corps inanimé d'une des Sirènes trouverait son repos ultime au golfe de Naples. ${ }^{5}$

Par des raisons multiples, un éventuel emploi autorefléxif et métapoétique du motif de Parthénopé ne peut pas être exclu. Sœurs lugubres des Muses, ${ }^{6}$ les Sirènes avec leur musique sinistre représentent une inspiration poétique étrange. Quant au nom parlant de la Sirène, par un jeu de mot fondé sur la ressemblance phonétique et sur le sens du mot grec $(\pi \alpha \rho \theta \dot{\varepsilon} v o \varsigma=$ jeune fille vierge $)$, Parthénopé est souvent mise en parallèle avec Parthenias qui est le sobriquet de Virgile. Ce rapport elliptique entre la Fille (la Sirène ou bien Perséphone, Kópๆ par excellence ${ }^{7}$ ) et le poète est souligné aussi par l'interprétation de M. Korenjak. À sa lecture, le nom de Parthénopé à la fin du chant IV serait préparé par le mot virgineum à la fin du chant I (429); leur ensemble encadrerait les Géorgiques par une allusion double à la mythologie grecque et à l'étymologie populaire du nom du poète. ${ }^{8}$

Selon le témoignage de la tradition biographique de l'Antiquité et du Moyen Âge, le nom Parthénias / Partenius / Partenose remonte aux mours du poète. Par un geste qui évoque l'intérêt étiologique de la poésie hellénistique, toutes les sources expliquent le sobriquet du poète par son excellence morale. ${ }^{9}$ Cependant, une note de Servius rarement examinée et très intéressante pour l'analyse actuelle, semble modifier l'admiration générale envers les mœurs purs virgiliens. Elle fait allusion à la pas-

\footnotetext{
${ }^{2}$ Servius ad Georg. IV 563 : Parthenope: id est Neapoli, quae primo ex corpore unius sirenis illic sepultae Parthenope est appellata.

${ }^{3}$ Le nom de Naples ne se lit pas chez Virgile à l'exception de Neapolis dans l'Etna pseudovirgilienne (430).

${ }^{4}$ Cf. Lyc. Alex. 712 sqq ; Strab. Géogr. 5. 4. 8; 6. 1. 1.

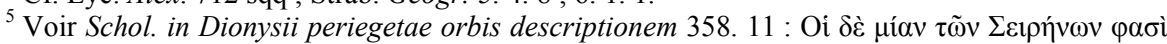

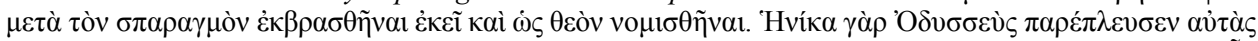

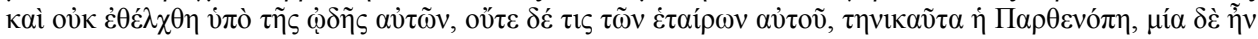



${ }^{6}$ Pour l'idée des Jenseitsmusen voir EGELER, M. : Walküren, Bodbs, Sirenen. Gedanken zur religionsgeschichtlichen Anbindung Nordwesteuropas an den mediterranen Raum. Berlin - New York 2011, pp. 407-408.

${ }^{7}$ Selon Apollonios de Rhodes (Arg. IV 896) les Sirènes sont les compagnonnes de Perséphone avant son enlèvement.

${ }^{8}$ Voir KORENJAK, M. : Parthenope und Parthenias: zur Sphragis der Georgika. Mnemosyne 48 (1995) pp. 201-202. Pour un jeu de mot fondé également sur l'idée de la virginité (Én. X 747) voir CuCCHIARELLI, A. : Vergil on killing Parthenius (Aen. 10. 748). ClJ 97 (2001) pp. 51-54.

${ }^{9}$ Cf. Serv. Vita Verg. 1: adeo autem verecundissimus fuit, ut ex moribus cognomen acciperet; nam dictus est Parthenias, omni uita probatus. Cf. Vita Monacensis II : fuit enim excellenti ingenii et tantae admiracionis quod ab omnibus Parthenius diceretur, id est bene tenens vel perciens, vel a Partenose, id est cum virtute probatus. Les vitae sont citées de l'édition de ZIOLKOWSKI, J. M. - PUTNAM, M. C. J. : The Virgilian Tradition: The First Fifteen Hundred Years. New Haven - London 2008, p. 203, 270.
} 
sion érotique du poète, vue comme un pouvoir nocif qui s'oppose nettement à l'idée de l'innocence suggérée par l'étymologie grecque du sobriquet latin: uno tantum morbo laborabat; nam inpatiens libidinis fuit. ${ }^{10}$ L'idée de l'amour fait partie intégrante aussi du mythe des Sirènes. ${ }^{11}$ La force irrésistible de cette voix féminine est inséparable de la séduction érotique qui ne met en danger que des hommes, comme l'illustre le récit d'Apollonios de Rhodes. Sans parler directement de la mort des Sirènes, l'auteur des Argonautiques place leur échec dans une couche plus profonde du mythe : la voix des filles serait réduite au silence dans un étrange concours musical dominé par la violence et la dissonance, où le phorminx d'Orphée essaie de violer la sonorité des vierges. $^{12}$

La possibilité d'un rapport particulier entre Virgile et la Sirène est confirmée aussi par la tradition locale. Ainsi, l'ancienne Parthénopolis, refondée et renommée Néapolis ${ }^{13}$ par l'ordre d'Octavien, vainqueur d'Actium, deviendrait un refuge pour le poète qui s'y retire pour vivre inaperçu. Selon le témoignage de Suetonius (Vita Verg. 11), le sobriquet Parthenias remonterait également à cet endroit : cetera sane vitae et ore et animo tam probum constat, ut Neapoli Parthenias vulgo appellatus sit. En plus, le séjour napolitain de Virgile peut être vu aussi comme un détachement conscient de la Rome d'Auguste, comme l'illustre une lettre de l'empereur qui s'indigne car Virgile s'enfuit de la ville de la Campanie à son arrivée. ${ }^{14}$ En suite, on doit mentionner aussi la similarité des cultes locaux de Parthénopé et de Virgile, transfiguré dans la mémoire collective des siècles en personnage mythique aux capacités surnaturelles qui sont mises en action en faveur de la ville. ${ }^{15}$ Toute comme celle de la Sirène, la dépouille du poète subit une translatio : elle est transportée de Brindisi, lieu de mort à Naples. Donc, l'autoportrait de l'auteur des Géorgiques en homme nourri par la maîtresse grecque d'un chant irrésistible et létal semble attribuer à ce passage une importance métapoétique qui est soulignée aussi par le téléstiche otia. ${ }^{16}$

${ }^{10}$ Pour le motif de Virgile impatiens libidinis voir ZIOLKOWSKI - PUTNAM (n. 9) pp. 203, 231, 307, 399, 713.

${ }^{11}$ Pour l'idée de la Sirène comme erotic women par excellence voir MILES (n. 1) p. 293, n. 35. On note qu'une certaine Parthénopé est la protagoniste tragique d'un roman grec érotique, voir HÄGG, TH. UtAs, B. : The Virgin and her Lover: Fragments of an Ancient Greek Novel and a Persian Epic Poem. Leiden 2003, pp. 46-48.



${ }^{13}$ Les sources voir MiLETTI, L. : Setting the Agenda: The Image of Classical Naples in Strabo's Geography and Other Ancient Literary. In Hughes, J. - BuOngiovanni, Cl. (eds.) : Remembering Parthenope: The Reception of Classical Naples from Antiquity to the Present. Oxford 2015, pp. 19-39, en particulier p. 24, n. 8 .

${ }^{14}$ Epist. 35. Malcovati : excucurristi a Neapoli. Pour les détails voir THOMAS, R. F. : Virgil and the Augustan Reception. Cambridge 2001, pp. 38-40.

${ }^{15}$ Voir les miracles de Virgile dans la Cronaca di Partenope, ZiolKowSKI - PUTNAM (n. 9) pp. 945-953. Voir aussi ABBAmOnTE, G. : Naples - A poet's City. Attitudes towards Statius and Virgil in the Fifteenth Century. In HuGHES - BUONGIOVANNI (n. 13) pp. 170-88.

${ }^{16}$ Pour le téléstiche otia (IV 563-565) voir NELIS, D. : Vergils' Library. In FARRELL, J. - PUTNAM, M. C. J. (eds.) : A Companion to Vergil's Aeneid and Its Tradition. Chichester - Malden MA, pp. 11-25, ici p. 21 ; DANIELEWICZ, J. : Vergil's certissima signa reinterpreted: The Aratean lepte-acrostic in Georgics I. Eos 100 (2013) pp. 287-295. 
Néanmoins, en outre de la musicienne redoutable du mythe, les vers virgiliens pourraient évoquer aussi un personnage historique, Parthénios de Nicée, vu traditionnellement comme médiateur de la culture grecque pour Virgile et ses amis. Parthénopé est bien présente aussi dans l'histoire de Parthénios : selon la tradition biographique, c'est la ville de Parthénopolis qui donnerait lieu à la première rencontre du futur poète et du Grec lettré. ${ }^{17}$ Dans l'étude qui suite, nous voudrions proposer quelques notes supplémentaires en faveur d'une interprétation qui suppose la présence d'une allusion métapoétique pas encore remarquée dans la sphragis des Géorgiques. À notre hypothèse, l'évocation de Parthénopé peut être considérée comme un énoncé autorefléxif sur l'inspiration poétique. Celui semble se fonder sur le renouvellement virgilien de la tradition de la tradition (avec une Sirène dans le rôle de la Muse) tout en établissant un hommage à Parthénios et particulièrement à ses Erotica Pathémata, à ce recueil des mythes inédites sur l'amour dont les réminiscences seraient détectables dans les allusions mythologiques du poème sur agriculture. Un autre passage rarement aperçu peut justifier la supposition d'un tel jeu métapoétique : la formule Parthenios circumdare saltos des Bucoliques (X 57) semble également rappeler les liens personnels et littéraires entre Gallus, Virgile et Parthénios. ${ }^{18}$

Parthénios est un auteur à la frontière de la littérature grecque et de romaine, et aussi à celle de la république tardive et de la monarchie. Selon sa biographie lacunaire qui ne permet que des conjectures, il est arrivé à Rome vers 73 av. J.-C. comme prisonnier des guerres mythridatiques. Libéré grâce à son érudition, ${ }^{19}$ le poète renommé s'intègre rapidement aux cercles littéraires romains, il entretient de bonnes relations avec les philhellènes de l'époque comme Catulle, Cinna, Luculle et les jeunes Cornelius Gallus et Virgile, écartés de lui par une génération. ${ }^{20}$ Tout en gardant son indépendance personnelle et ses préférences esthétiques, Parthénios dans son rôle de grammaticus qui lui est attribué par la tradition tardive, ${ }^{21}$ paraît avoir collaboré comme expert littéraire chez les auteurs de la nouvelle vague de la poésie latine, sans pouvoir parler d'un enseignement de la langue grecque aux adolescents débutants dans la poésie. ${ }^{22}$

Les avis concernant son rôle dans la transmission de la culture hellénistique et sur l'influence de son œuvre se divergent largement. ${ }^{23}$ Dans la recherche antérieure initiée au XIX ${ }^{\text {ème }}$ siècle, il a été vu comme auteur sinequanon du renouvellement poétique,

${ }^{17}$ Sur cette question disputée voir LightFoOt, J. L. : Parthenius of Nicaea. The Poetical Fragments and the Erotika pathemata. Oxford 1999, p. 14 et CALDERÓN DORDA, E. : Partenio, maestro de Virgilio. In Simposio virgiliano conmemorativo del bimilenario de la muerte de Virgilio. Murcia 1984, pp. $217-223$.

${ }^{18}$ Voir HutChInSON, G. O. : Greek to Latin: Frameworks and Contexts for Intertextuality. Oxford 2013, p. 16.



${ }^{20}$ Pour une esquisse biographique voir LIGHTFOOT (n. 17) pp. 9-16 et FranCESE, CH. A. : Parthenius of Nicaea and Roman Poetry. Frankfurt 2001, pp. 17-29. Pour une synchronisation possible avec Virgile et Gallus voir FRANCESE pp. 23-27. Sur la relation entre Virgile et Parthénios voir LIGHTFOOT (n. 17) pp. 14-16, 70, 165, 168-189.

${ }^{21}$ Cf. Macr. Sat. V 17. 18 (T 9a Lightfoot), voir aussi LiGHTFOOT (n. 17) p. 85.

${ }^{22}$ Pour la révision du concept de Parthenios grammaticus voir FRANCESE (n. 20) pp. 37-43.

${ }^{23}$ Voir les comptes rendus chez LightFOOT (n. 17) pp. 50-76 et FrANCESE (n. 20) pp. 9-47. 
comme celui qui fait découvrir aux Romains Callimaque ${ }^{24}$ et Euphorion, les modèles les plus imités par les poetae novi. ${ }^{25} \mathrm{Un}$ autre axe d'études voulait mettre en évidence l'emprise définitive de son Areté, épicédie à la mémoire de sa femme chez les auteurs de l'élégie latine, en particulier chez Gallus. ${ }^{26}$ Cependant, l'omnipotence de Parthénios a été sérieusement mise en doute par plusieurs selon qui la vogue de la littérature

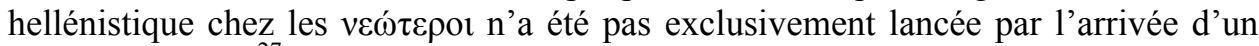
seul Grec érudit. ${ }^{27}$ Quant à sa qualité littéraire, les fragments de son œuvre lyrique presque totalement disparue ne rendent pas possible d'énoncer un avis définitif, malgré les découvertes papyrologiques récentes. ${ }^{28}$ Les Erotica Pathémata, son ouvrage unique transmis dans son intégrité aurait de ce point de vue une importance primordial. Il paraît que Parthénios, malgré son anima naturaliter Callimachea ${ }^{29}$ propose avec ce recueil une œuvre qui par son goût particulier, par son approche inouïe de la mythologie se diffère notamment de l'esthétique callimaquénne.

Au lieu de faire des hypothèses sur le rapport de Parthénios et des épyllia également disparus de Cinna, de Calvus, de Gallus, dans la suite nous proposons l'examen de deux textes intègres, celui des Erotica Pathémata et des Géorgiques. On concède, la comparaison etre un recueil de synopsis rédigé dans un style à première vue simple et un chef-d'œuvre limé à la perfection peut être étonnante. Pourtant, le deux ouvrages paraissent se converger aux certains points ce qui sollicite un examen détaillé pas encore fait auparavant. ${ }^{30}$

Par sa lettre dédicatoire, la collection parthénienne est adressée à Gallus qui devrait puiser à ce petit aide-mémoire des idées pour ses compositions littéraires.

${ }^{24}$ Sur sa poésie en général voir LightFoot (n. 17) pp. 16-49. Pour la communauté des idées esthétiques de Parthénios et de Callimaque voir T 5, 6 Lightfoot ; FANTUZZI, M. - HUNTER, R. : Muse e modelli. Roma-Bari 2002, p. 336.

${ }_{25}^{25}$ Pour un compte rendu voir FrANCESE (n. 20) p. 10.

${ }^{26}$ Voir GÄRTNER, TH. : Überlegungen zur Genese der römischen Liebeselegie aus der hellenistischen Dichtung. AAntHung 46 (2006) pp. 213-237.

${ }^{27}$ Voir CROWTHER, N. B. : Parthenius and Roman poetry. Mnemosyne 29 (1976) 65-71 ; FANTUZZI - HUNTER (n. 24) p. 537.

${ }^{28}$ Pour les fragments de ses Metamorphoses (avec les mythes d'Adonis, d'Astarte et de Narcissus) voir HuTCHINSON, G. O. : The Metamorphosis of Metamorphosis : P. Oxy. 4711 and Ovid. ZPE 155 (2006) pp. 71-84 ; MAGNELLI, E. : On the new fragments of Greek poetry from Oxyrhynchus. ZPE 158 (2006) pp. 9-12.

${ }^{29}$ Formule d'A. Cameron citée par LightFoOt (n. 17) p. 17.

${ }^{30}$ Sans proposer une revue exhaustive, on rappelle quelques passages virgiliens dont l'éventuelle origine parthénienne est signalée par les travaux antérieures. B. OTIS (Virgil: A Study in Civilized Poetry. Oxford 1964, pp. 138-140) remarque l'origine parthénienne de la Bucolique VI 31-81 et des Géorg. IV 345-47. Sans entrer dans les détails, CALDERON DORDA suppose une influence primordiale sur toute l'œuvre (n. 17). Chez LightFOOT (n. 17, p. 195), le seule passage des Géorgiques emprunté avec certitude à Parthénios serait I 437, l'éventuelle origine parthénienne des mythes de Daphné et de Scylla dans les Géorgiques ne sont que de pure conjectures (ibid. pp. 165, 297, 561). Pour le rapport entre fr. 10 Lightfoot et Én. IV 345 voir ibid. pp. 150-151. Dans le recueil d'article très important édité par A. ZUCKER (Littérature et érotisme dans les Passions d'amour de Parthénios de Nicée. Actes du colloque de Nice, 31 mai 2006. Grenoble 2008) le nom de Virgile ne se lit que deux fois (p. 11 : Parthénios comme maître de Virgile, p. 196 : l'histoire d'(Enone chez Ovide). En examinant l'élaboration virgilienne des sources scientifiques grecques des Géorgiques, THIBODEAU, PH. (Playing the Farmer: Representations of Rural Life in Vergil's "Georgics". Berkeley - Los Angeles - London 2011, p. 281, n. 21) établit une analogie double entre Aristote et Virgile d'une part, Parthénios et Gallus d'autre part. 
Allié confident d'Octavien, Gallus joue un rôle important dans la guerre contre MarcAntoine, pour son activité politique et militaire il est honoré par une position prestigieuse. Plus tard, victime des accusations et des intrigues toujours mal connues, il est condamné à l'exil, exclu de l'ordre équestre et de la maison d'Auguste, avec tous ses biens confisqués. Son suicide en 26 av. J.-C. ne termine pas sa disgrâce : il est frappé d'une damnatio memoriae qui veut effacer sa mémoire définitivement. En ce qui concerne la fortune de Parthénios pendant la période augustéenne, elle n'est pas connue, ce qui est bien remarquable vu que les Césars de l'avenir l'estiment beaucoup : Tibère fait ériger sa statue dans les bibliothèques publiques, Hadrien, en imitant le culte des poètes grecs, fait renouveler sa tombe. ${ }^{31}$

En cherchant les éventuels points communs du recueil de Parthénios et des Géorgiques, c'est le problème des laudes Galli qui s'impose. Le poème didactique écrit entre 36 et 29 av. J.-C. a été longuement regardé comme le document primordial de la renovatio Augusti. Le changement politique, économique et idéologique initié par l'empereur veut redresser la société gravement blessée dans les guerres civiles et reconduire les Romains aux valeurs ancestrales, aux mos maiorum, tout d'abord par les travaux de champs. Or, selon le commentaire de Servius, la fin du livre IV qui était un hommage à Gallus dans la version originale a été remplacée par l'histoire d'amour d'Orphée et d'Eurydice par l'ordre d'Octavien. ${ }^{32}$ Bien que la fiabilité de cette information soit réfutée par des simples raisons chronologiques, la lecture allégorique du mythe final fait régulièrement apparition dans la recherche : Orphée ne serait que Virgile (ou bien Gallus), Aristée, l'apiculteur divin incarnerait Auguste, roi de la ruche romaine contemporaine. ${ }^{33}$

L'objectif de notre recherche n'est pas de reprendre le dossier sur la révision politiquement forcée du livre IV, encore moins de celui sur la relation entre Virgile et Auguste et de l'éventuel programme augustéen des Géorgiques, ${ }^{34}$ mais de reconsidérer les relations entre la représentation des passions érotiques chez Parthénios et chez le poète des Géorgiques. Il paraît que leurs ouvrages sont liés non seulement par le souvenir de Gallus, mais aussi par une certaine ressemblance thématique. On poserait plusieurs questions. Peut-on parler d'une inspiration parthénienne des allusions mythologiques des Géorgiques? Est-ce possible que les narrations érotiques grecques auraient une influence sur le concept d'amour du poème latin ? Par ses mythes cachés, Virgile répondrait-il à l'invitation poétique lancée par Parthénios, aux questions éthiques et littéraires à peine articulées dans les récits brefs des Erotica

${ }^{31} \mathrm{~T} 3$ et 4 Lightfoot.

${ }^{32}$ Serv. ad. Géorg. IV intr., ad Ecl. X 1. Pour une revue voir ThOMAS, R. F. (ed.) : Virgil, Georgics. Cambridge 1988, pp. 13-16. Pour le Corpus Servianum en général voir FowLER, D. : The Virgil Commentary of Servius. In MARTINDALE, CH. (ed.) : The Cambridge Companion to Virgil. Cambridge 1997, pp. 73-78. Servius dans ses phrases bien ambiguës concernant les traits antihérö̈ques de la représentation virgilienne laisse entendre aussi des voix opposées à l'idéologie officielle augustéenne. Pour l'herméneutique de son commentaire voir THOMAS (n. 14) pp. 93-121.

${ }^{33}$ Pour une lecture allégorique récente voir CADILI, L. : Viamque adfectat Olympo. Memoria ellenistica nelle «Georgiche» di Virgilio. Milano 2001.

${ }^{34}$ Voir le chapitre Virgil and August chez THOMAS (n. 14) pp. 25-54. 
Pathémata, tout comme on le suppose dans le cas d'Ovide $?^{35}$ En outre, les histoires d'amour de Parthénios et de Virgile se concentrent dans leur grand majorité absolue sur les forces irrépressibles et irrationnelles de la passion et elles s'achèvent sur les crimes de l'adultère, de l'infanticide, de l'inceste. ${ }^{36}$ Comment peut-on placer ces narrations virgiliennes nées dans l'otium napolitain dans le contexte morale de l'époque augustéenne qui met en relief les valeurs civiques de la famille, du mariage, d'une vie privée sage, sombre et responsable, fondée sur l'idée du negotium ?

Pour aborder ces problèmes, il vaut mieux commencer par les caractéristiques généraux des deux œuvres. Rédigés quelque temps entre 52 et 26 av. J.-C., les Erotica Pathémata contiennent les trames de 36 mythes érotiques dans une forme condensée et économique. En concentrant sur l'action, Parthénios évite les digressions, les descriptions, les dialogues et il s'oriente vers une conclusion rapide. ${ }^{37}$ Ses récits sont empruntés surtout à la poésie et à l'historiographie hellénistiques, ses mythes se trouvent souvent à l'envergure des grands sujets épiques de la tradition homérique. ${ }^{38}$ Dans une approche de la mythographie, le concept général du recueil conforme parfaitement avec les traits généraux des mythes grecs qui, faute d'un corpus officiel, ne vivent que dans les allusions littéraires. Cependant, ils ne sont jamais expliqués dans leurs intégrité par les œuvres poétiques afin de fournir une version définitive et officielle d'une histoire, ce qui permet de les redire à l'infini, en expliquant et en créant des nouvelles allusions quelques fois surprenantes. ${ }^{39}$

La collection parthénienne, mise en ensemble par un intérêt philologique pas trop rigoureux, veut fournir à Gallus des idées à élaborer dans des futurs poèmes épiques et élégiaques, ${ }^{40}$ en proposant, peut-être, aussi un modèle stylistique. D'autre part, Parthénios grammaticus semble aider son ami (ou bien son amoureux $?^{41}$ ) dans l'interprétation des passages poétiques obscurs. Enfin, il peut divertir son lecteur éventuellement intéressé par les sujets lascifs, bizarres ou carrément pervers. Le caractère littéraire du recueil, mis en doute souvent dans le passé, a été revendiqué surtout par les travaux importants des chercheurs français. Leurs examens de la structure et des stratégies narratives donnent des arguments solides en faveur d'une composition rédigée par des principes esthétiques notables. Ce qui auparavant a été considéré comme

${ }^{35}$ Voir les études de FABRE-SERRIS, J. : Histoires d'inceste et de furor dans les Métamorphoses 9 et dans le chant en catalogue d'Orphée : une réponse d'Ovide au livre 4 des Géorgiques. Dictynna 2 (2005) http://dictynna.revues.org/12 ; FABRE-SERRIS, J. : Ovide lecteur de Parthenios de Nicée. In ZUCKER (n. 30) pp. 189-205.

${ }^{36}$ Sur l'importance du motif de l'inceste voir Billault, A. : La littérature dans les Erotica Pathémata de Parthénios. In ZUCKER (n. 30) pp. 13-26, en particulier p. 23.

${ }^{37}$ Cf. SpataforA, G. : Les Erotica Pathémata de Parthénios et la réécriture en format abrégé. In ZUCKER (n. 30) pp. 27-38.

${ }^{38}$ Pour le contexte homérique voir Billault (n. 36) pp. 17-18 et SistakOU, E. : Reconstructing the Epic. Cross-Readings of the Trojan Myth in Hellenistic Poetry [Hellenistica Groningana 14]. Leuven 2008 p. 136.

${ }^{39}$ Voir CAMERON, A : Greek Mythography. Oxford 2004, pp. 237-238.

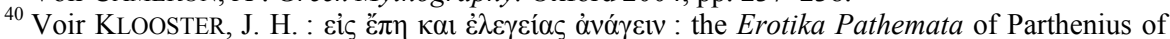
Nicaea. In BAUMBACH, M. - BÄR, S. (ed.) : Brill's Companion to Greek and Latin Epyllion and Its Reception. Brill's companions in classical studies. Leiden-Boston 2012, pp. 309-332.

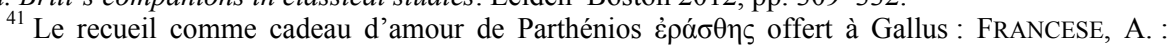
L'érotisme dans les Erotica Pathémata de Parthénios. In ZUCKER (n. 30) pp. 163-174. 
un opuscule modeste ayant une ambition littéraire minimale, ${ }^{42}$ comme un conglomérat de récits sans un plan général logique, ${ }^{43}$ est désormais regardé comme une composition organisée, qui représente l'esthétique de l'ellipse et de la varietas et qui sollicite la collaboration d'un lecteur attentif. ${ }^{44}$ Ce qui a été vu comme un texte plat, sec et mal soigné semble dorénavant être un poème en prose. ${ }^{45}$

Quant au regard parthénien sur les mythes, le narrateur les fait voir dans une optique particulière qui se détache consciemment de la tradition héroïque. ${ }^{46}$ Tout en empruntant ses personnages au mythe de Troie (Achille, Ulysse, Paris) ou à l'histoire grecque (Périandre, Croesus), il passe sous silence les guerres, les conquêtes et d'autres entreprises viriles afin de se concentrer sur les émotions, sur les enjeux psychologiques du désir érotique. Il scrute l'effet de la passion sur l'âme humaine en représentant des crimes effrayants ou des comportements anormaux qui ne sont presque jamais commentés par une éthique autoritaire. Par ses topoï narratifs liés étroitement aux problèmes moraux de la conduite érotique ${ }^{47}$ et par sa forme abrégée, le recueil serait une expérience remarquable de la réécriture, un texte médiateur entre des genres divers. ${ }^{48}$

La relation entre les deux auteurs concernés par notre examen est inséparable de la tradition déjà mentionnée selon qui Parthénios était le maître de Virgile en grec. Tout en partageant la révision du mot grammaticus chez Francese et l'avis de Crowther selon qui une tutelle culturelle ne s'achève pas forcément sur une influence poétique immédiate, ${ }^{49}$ on doit rappler que les notes d'Aulu-Gelle et de Macrobe écrites à la liste miniature des dieux maritimes au chant I 437 des Géorgiques ${ }^{50}$ témoignent d'un emprunt délibéré à Parthénios. Celui serait motivé par une intention d'aemulatio mise en évidence aussi par l'acrostiche du nom de Virgile. ${ }^{51}$

\footnotetext{
${ }^{42}$ Cf. CAMERON (n. 39) p. 272.

${ }^{43}$ L'unité thématique du recueil est suggérée aussi par CADILI (n. 33) p. 112 et CAMERON (n. 39) p. 301.

${ }^{44}$ Sur la structure générale du recueil et sa tendance à disjoindre les éléments cohérents voir VoISIN,
} D. : Dispositio et stratégies littéraires dans les Erotica Pathémata de Parthénios. In ZuCKER (n. 30) pp. $39-65$.

${ }^{45}$ Voir BIRAUD, M. : Échos imitatifs et transgressifs des poètes alexandrins dans les Erotica Pathémata 11 et 34 de Parthénios de Nicée. In VIAL, H. (éd.) : La Variatio. L'aventure d'un principe d'écriture. Paris 2014, pp. 103-115.

${ }^{46}$ Voir FRANCESE (n. 20) pp. 69-73.

${ }^{47}$ Pour les topoï les plus importants (initiation sexuelle, trahison, inceste, viole, adultère, suicide, enfant naturel, sacrifice d'enfants, remords, etc.) et pour la technique de lier les histoires voir VoISIN (n. 44) passim.

${ }_{48}^{48}$ Pour l'influence du roman et de la tragédie voir Billault (n. 36) pp. 21-23.

${ }^{49}$ Voir CROWTHER (n. 27) p. 71.

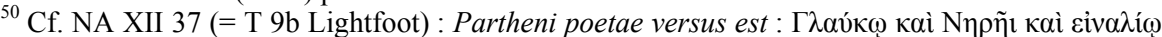

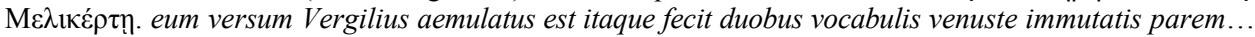
cf. Macr. Sat. V 17. 18 (T 9a) : Versus est Parthenii, quo grammatico in Graecis Virgilius usus est: Glauco et Panopeae et Inoo Melicertae. - Sur la source des notes d'Aulu-Gelle et de Macrobe voir LIGHTFOOT (n. 17) pp. 84-85.

${ }^{51}$ Pour l'éventuel acrostiche PU - VE - MA dans les vers 429-431-433 voir NeLIS (n. 16) p. 22. Le passage est vu par HUTCHINSON (n. 18) p. 305 comme le document de l'émancipation poétique de Virgile qui s'éloigne de la tradition hésiodique par l'aide d'Arate (cf. I 351) et de Parthénios. Voir aussi MORGAN, L. : Patterns of Redemption in Virgil's Georgics. Cambridge 1999, pp. 22, 40. 
Avant d'analyser la communauté des motifs de deux compositions, on doit s'attarder sur une autre remarque d'Aulu-Gelle qui concerne les principes virgiliens de la réécriture et la nature sélective de son imitation. Le poète romain, dit-il, au lieu d'une traduction mot-à-mot enlève des passages de ses modèles grecs ou bien il y ajoute des éléments nouveaux. ${ }^{52}$ De notre point de vue, cette flexibilité est bien important. À l'exception du catalogue mentionné des dieux de la mer mentionné ci-dessus, dans le poème sur l'agriculture ne se trouvent ni des citations ni des évidences lexiques qui pourraient être considérées comme des citations ou bien des imitations directes de Parthénios. Ainsi, dans la suite nous nous concentrons sur des concordances thématiques, des motifs qui occurrent similairement chez les deux auteurs. Pour valider cette méthode, on se réfère à l'étude de Clausen ${ }^{53}$ qui, malgré l'absence des correspondances verbales, argumente avec justesse en faveur de l'origine parthénienne de la représentation de la mort de Didon au chant IV de l'Énéide. La reine de Carthage se suicide entourée par des objets personnels d'Énée. Au chapitre II des Erotica Pathémata, c'est la jolie Polymèle séduite par le perfide Ulysse qui se prête à mourir en serrant dans les mains un objet qui provient du butin troyen (et qui, visiblement, doit être défini par le choix poétique de Gallus ou bien de n'import quel nouveau narrateur du récit dans l'avenir). La vie de la fille est sauvée par l'amour incestueux de son frère, l'histoire se termine dans un mariage heureux entre frère et sœur, ce qui s'oppose nettement au destin tragique de Didon.

Ce n'est pas le seul passage de l'épopée qui remonterait à un modèle parthénien. À notre avis, un épisode du chant VIII pourrait être considéré également comme la réécriture d'un mythe de Parthénios effectuée par la technique de Kontrastimitation. Hercule, en retour de l'Ouest avec son troupeau volé de Géryon, est attardé en Italie par Cacus qui lui prend les bœufs et les enferme dans un antre (VIII 185-275). Pour les récupérer, le héros doit descendre au fond de la terre et se battre avec le monstre chthonien. Un récit similaire se lit dans une narration étiologique de Parthénios ( $E P$ $\mathrm{XXX}$ ) où l'itinéraire d'Hercule est légèrement différent. Le héros avec ses vaches fait un trajet vers le Nord où Keltiné la jolie fille rusée du roi Bretannos, lui cache ses animaux. Afin de les reprendre, il doit faire l'amour avec la princesse (EP XXX 18-19

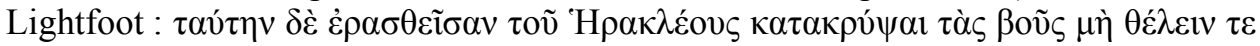

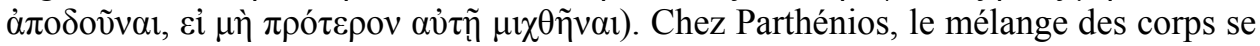
réalise dans un rapport érotique qui donne naissance au héros éponyme des Celtes ; chez Virgile, il est transformé dans une bataille des forces cosmiques transposée dans l'au-delà. À ma connaissance, Parthénios n'est jamais mentionné parmi les sources possibles de l'épisode virgilien. ${ }^{54}$

Les éventuelles analogies et oppositions entre les narrations de Parthénios et les allusions mythologiques des Géorgiques sont beaucoup moins connues. Tout

\footnotetext{
${ }^{52}$ Cf. NA IX 9.3 (= T 9c Lightfoot) : Vergilius cum aut Homeri, aut Hesiodi aut Apollonii aut Parthenii aut Callimachi aut Theocriti aut quorundam alios locos effingeret, partim reliquit, alia expressit.

${ }^{53}$ Voir ClAUSEN, W. : Virgil and Parthenius. HSCPh 80 (1976) p. 179.

${ }^{54}$ Voir par exemple GALINSKY, K. G. : The Hercules-Cacus Episode in Aeneid VIII. TAPhA 87 (1966) pp. 18-51 ; SuTTON, D. : The Greek Origins of the Cacus Myth. CQ 27 (1977) pp. 391-393. PUTNAM, M. C. J. qui suppose dans l'épisode une insinuation à caractère sexuel ne mentionne pas Parthénios, voir Virgil's Aeneid: Interpretation and Influence. Chapel Hill - London 1995, pp. 31-32.
} 
d'abord, à propos de l'amour, la recherche virgilienne se concentre presque uniquement sur l'histoire d'Orphée et d'Euridyce à la fin du chant IV. L'épisode est généralement vu comme un épyllion à l'inspiration hellénistique dont le ton tragique est quelquefois mis en parallèle avec les récits de Parthénios ${ }^{55}$ (bien que le musicien de Thrace soit nulle part mentionné dans les Erotica Pathémata où l'art visuel, la musique ou la littérature ne sont jamais thématisés). Pourtant, il nous semble qu'à côté du chant III qui parle directement de la procréation et du comportement sexuel des animaux, dans le poème consacré aux sujets pratiques de travaux agraires se cacheraient plusieurs morceaux étroitement liés à la notion d'amour et aux problèmes éthiques de vivre une passion. ${ }^{56}$

Les images naturelles, la représentation virgilienne des plantes, des bêtes dans leur majorité semblent se référer de façon elliptique aux mythes d'origine où le désir érotique serait la motivation primordiale des événements. La possibilité d'une telle lecture des Géorgiques est confirmée avant tout par le commentaire de Servius dont les notes écrites à ces passages sont dominées par des récits érotiques. L'emploi des références étiologiques aurait un rôle primordial afin d'humaniser le monde du poème qui, sans compter le personnage plutôt abstraite de l'arator, serait un cosmos imparfait à cause de l'absence de l'homme. La série de ces allusions mythologiques qui s'épanouit dans l'histoire d'Orphée, développée dans une ampleur remarquable, paraît établir en filigrane une deuxième charpente du poème qui est moins visible au rapport des thèmes majeurs de quatre livres. Parmi ces réminiscences mythologiques on constate facilement la dominance des allusions qui concernent les amours des dieux et des mortels dans toute leur variété. Le paysage des Géorgiques est animé par des êtres jadis humains qui sont métamorphosés en arbres, en fleurs, en étoiles, en montagnes ou bien qui sont enfermés dans des corps d'animaux. Toutes ces transfigurations se réalisent par la pitié des immortels, ou, encore plus souvent par la colère des divinités jalouses et impitoyables.

Voyons désormais quelques exemples, sans prétendre d'analyser de façon exhaustive tous les cas où la présence des éventuels liens thématiques entre les Erotica Pathémata et les Géorgiques peut être supposée. Le calendrier agricole du chant I représente les tâches champêtres distribuées en raison du changement des saisons. À propos des oiseaux qui signalent l'arrivée de la période des pluies, les vers 406-409 évoquent le mythe de Nisus et de Scylla, très à la vogue dans la poésie après-virgilienne ${ }^{57}$ qui

\footnotetext{
${ }^{55}$ Voir Wilkinson, L. P. : The Georgics of Virgil: A Critical Survey. Cambridge 1969, p. 184 ; THOMAS (n. 32) I p. 15, n. 41 ; MORGAN (n. 51) pp. 162-164, 169.

${ }^{56}$ Pour un examen détaillé voir PATAKI, E. : Métamorphoses, amours et abeilles. Notes sur le livre IV des Géorgiques. AAntHung 44 (2004) pp. 263-82.

${ }^{57}$ Servius ne commente le mythe qu'à propos d'Éqlogue VI 74 : Scylla fuit Nisi, Megarensium regis, filia. contra quos dum, devictis iam Atheniensibus, pugnaret Minos propter filii Androgei interitum, quem Athenienses et Megarenses dolo necaverant, adamatus a Scylla est, Nisi filia, quae ut hosti posset placere, comam purpuream parenti abscisam ei obtulit, quam Nisus ita habuerat consecratam, ut tamdiu regno potiretur, quamdiu illam habuisset intactam. postea et Scylla, a Minoe contempta, vel dolore, quod contempta esset, vel (quod) quasi parricida a Minoe ad puppim religata tracta sit, in avem Cirim conversa est, et Nisus extinctus deorum miseratione in avis mutatus est formam: quae aves hodie, ut ipse in georgicis docet, flagrant inter se magna discordia.
} 
illustre le pouvoir destructif de l'amour. La princesse, éprise de l'ennemi qui attaque son pays, dans sa folie amoureuse lui livre son père, mais finalement elle est dédaignée par l'étranger. Virgile ne doit pas raconter l'histoire en détail à son publique qui la connaît bien. ${ }^{58}$ On ne voit qu'une image zoologique, père et fille transformés en oiseaux qui tout de même gardent leur hostilité.

Le mythe de Scylla ne se lit pas dans les Erotica Pathémata (or, il a été traité par les Métamorphoses de Parthénios ${ }^{59}$ ). Évidemment, une origine parthénienne directe de l'épisode chez Virgile, rarement supposée dans les études, ${ }^{60}$ ne peut pas être prouvée avec certitude. Cependant, le topos narratif d'un conquérant qui est adoré par la fille de son ennemi et qui s'en profite de façon malhonnête afin de réaliser son plan de guerre, figure plusieurs fois dans le recueil parthénien, ainsi à la séquence des chapitres XXI-XXII. ${ }^{61}$ Le déroulement des événements est plus ou moins identique : une fille royale devient déloyale à sa famille pour gagner le cœur d'un étranger dangereux. Plus tard, trompée dans ses illusions et abandonnée, elle doit mourir victime de la vengeance de sa famille ou bien tuée par l'amant infidèle. Dans le chapitre 21 qui remonte à un mythe lesbien (dont la version écrite par Apollonios de Rhodes est longuement citée), c'est Achille qui ne tient pas sa parole donnée à Peisidiké. ${ }^{62}$ Selon le récit parthénien marqué par un langage homérisant et un ton fort pathétique, Achille, après avoir réalisé sa conquête militaire par l'abus de la confiance de la fille naïve, au lieu de la marier la fait lapider par ses soldats. Le récit suivant (EP XXII) évoque la siège de Sardeis où Cyrus, attaqueur de la ville rompe son serment prêté à Nanis, fille du roi Croesus qui tombe amoreuse de lui. L'aboutissement des événements, le destin de la princesse traîtresse cette fois ne sont pas racontés. Parthénios laisse ouverte sa narration en incitant son lecteur à la terminer par une fin doublement tragique (où la défaite de la patrie coïncide avec la mort violente de la princesse) ou bien par un dénouement un peu plus heureux. ${ }^{63}$ On est tout à fait d'accord avec les études qui mettent en relief le retour du motif de la trahison par amour dans le récit sur Tarpée dans l'élégie romaine de Properce (III 19). ${ }^{64}$ Cependant, il paraît que la fortune virgilienne

${ }^{58}$ À noter : à côté de Scylla transfigurée en oiseau, Virgile connaît aussi le monstre maritime homonyme qui n'est présenté que dans l'Énéide. Pour le jeu virgilien fondé sur des homonymes mythologiques voir Horsfall, N. : Virgil, Parthenius and the Art of Mythological Reference. Vergilius 37 (1991) pp. 31-36, surtout p. 35.

Cf. fr. 24 Lightfoot, pour les détails voir LiGHTFOOT (n. 17) pp. 164-167 et FRANCESE (n. 20)

${ }^{60}$ Voir GALE, M. : Virgil on the Nature of Things. The Georgics, Lucretius and the Didactic Tradition. Cambridge 2000, p. 128 , n. 38 (sans une analyse détaillée). À propos de la ressemblance stylistique et métrique entre l'histoire de Byblis (EP XI) et de la Scylla virgilienne voir FRANCESE (n. 20 ) p. 59.

${ }^{61}$ Les détails voir chez LIGHTFOOT (n. 17) pp. 496-507.

62 Pour l'ironie de l'onomastique parthénienne voir Billault (n. 36) p. 25.

${ }^{63}$ Une version moins tragique du motif de l'amour de l'ennemi est proposée par le récit sur Polycrité ( $E P$ IX). Cette fois c'est la fille patriote qui profite de l'amour fou du chef de l'armée hostile afin de sauver sa ville. Néanmoins, elle est victime de sa gloire : brave et rusée, elle est suffoquée par le poids des cadeaux d'honneur de ses compatriotes.

${ }^{64}$ Pour la réécriture du mythe de Scylla dans le Ciris et chez Ovide et pour son rapport avec les récits sur Tarpée chez les élégiaques latins voir avant tout DELBEY, E. : Aimer son ennemi(e). In ZUCKER (n. 30) pp. 175-188, voir aussi FRANCESE (n. 20) pp. 157-183. 
du motif ne serait pas négligeable non plus, comme l'illustre une autre variante de la même trame narrative.

Cette allusion elliptique à un mythe d'amour (d'ailleurs, bien remarquable par sa fin heureuse qui est un phénomène exceptionnel chez Virgile) se lit également au chant I des Géorgiques. Le vers 222 Cnosiaque ardentis decedat stella Coronae rappelle le mythe d'Ariane. Passionnée pour Thésée, la Crétoise facilite l'assassinat de son demi-frère monstrueux. Après avoir tué le Minotaure, l'Athénien déloyal rejette l'amour de la fille. Au bord de se suicider, Ariane est sauvée par Dionysos qui l'enlève au ciel pour la marier, comme en témoigne la ceinture de la fiancée insérée dans les astres. ${ }^{65}$ Le mythe d'Ariane serait trop connu pour être raconté dans les Erotica Pathémata. Néanmoins, son cœur narratif montre une similarité notable avec des récits parthéniens qui abordent la perfidie érotique utilisée en faveur du succès d'une entreprise politique ou militaire. En ce qui concerne la nouveauté de la version virgilienne, elle s'impose premièrement par son point de vue entièrement féminin, ${ }^{66}$ deuxièmement par le motif du catastérisme, type narratif répandu dans la poésie étiologique de l'hellénisme (qui, par contre, est inconnu chez Parthénios et qui n'a pas non plus été abordé dans le carmen 64 de Catulle). Troisièmement, on doit remarquer l'intervention divine par laquelle l'histoire obtient une fin rassurante : trahie par un mortel, Ariane trouve sa félicité en épousant un dieu. En concentrant sur la transfiguration en étoile de la ceinture nuptiale, Virgile paraît vouloir mettre en relief un détail du mythe qui serait moins connu que la ruse du fil d'Ariane ou le chagrin de la fille abandonnée sur Naxos. Cette intention de Virgile de transmettre les moments oubliés ou pas encore dits du mythe grec concorde d'une part avec son programme poétique articulé dans le prologue du chant III. Ainsi, au lieu de répéter des thèmes surannés, le poète veut proposer quelque chose de nouveau aux oreilles oisives des lecteurs latins (III 3 : cetera, quae vacuas tenuissent carmine mentes ${ }^{67}$ ). D'autre part, son attitude paraît rappeler le principe esthétique d'obscur, d'ignoré et du non-dit qui semble être un trait caractéristique général des Erotica Pathémata. (Nonobstant, l'aboutissement heureux de la version virgilienne avec une Ariane divinisée se diffère notamment de l'atmosphère souvent effrayante des narrations parthéniennes.)

L'idée de la protection divine est présente chez Virgile aussi dans une allusion qui rappelle l'origine humaine des martins-pêcheurs, oiseaux privilégiés par la déesse de la mer (I 398) : Dilectae Thetidi alcyones. L'aition transmis par Servius voit dans les oiseaux Céyx et sa femme, Alcyoné qui méritent le respect de Thetis par leur

${ }^{65}$ Voir Serv. ad Georg. I 222 : Theseus cum ad interimendum Minotaurum Cretam venisset, miseratam eius Ariadnen auxilium ei ad occidendum filo ducto Minotaurum praestitisse; Theseum vero Ariadnen, utpote salutis auctorem, secum qui cum ad Naxum insulam delati essent, Ariadnen ibi vel consulto vel necessitate vel monitu Mercurii a Theseo derelictam. quam cum Liber pater adamasset, coronam ei dempto pudore, sicut supra dictum est, dicitur obtulisse: quam etiam inter sidera postea conlocavit.

${ }^{66}$ Ariane n'est jamais ailleurs évoquée chez Virgile. Thésée est mentionné dans les Géorgiques (II 383) uniquement dans le contexte du théâtre athénien, dans l'Énéide à propos de sa catabase (VI 122 , 393, 618).

${ }^{67}$ Pour le sens disputé de vacuas mentes voir par exemple PUTNAM, M. C. J. : Italian Virgil and the Idea of Rome. In VOLK, K. (ed.) : Vergil's Georgics. Oxford 2008, pp. 146-147. 
fidélité conjugale. ${ }^{68}$ Le couple n'est pas mentionné chez Parthénios. Cependant, sans être conjoint avec l'idée de la métamorphose, le motif d'un amour tragique vécu dans les cadres légitimes du mariage fait partie du recueil, par exemple dans le mythe de Rhésus qui est la dernière narration parthénienne (EP XXXVI) et qui figure aussi dans les Géorgiques (IV 462, à voir plus tard). Pour retourner à Céyx et à son épouse, on doit noter une étrange similarité des noms propres qui pourrait servir de base pour un jeu de mot délibéré, tellement typique chez Virgile. Dans le chapitre 27 des Erotica Pathémata le lecteur fait connaissance de l'histoire d'une certaine Alcynoé ${ }^{69}$ qui montre des traits de caractère plainement différents de ceux d'Alcyoné. Femme mariée, Alcynoé chasse de sa maison une servante bien dévouée sans la payer, ce qui met en colère Athéné. La déesse indignée réalise une vengeance tout à fait particulière. Alcynoé doit éprouver une passion folle pour un étranger venu d'outre-mer qui l'amènerait chez lui. Or, à mi-chemin elle est prise d'une vague de remords qui l'incitent à se suicider : la repentie se jette dans la mer, son geste évoque le rite purificatoire du katapontisme. Le motif de l'amour comme punition d'un acte injuste ne revient pas dans le recueil grec ni chez Virgile.

Le chant II qui traite les soins des plantes n'abonde pas en allusions étiologiques. ${ }^{70}$ Le seul vrai mythe d'origine où l'intention poétique de l'auteur est évidente se montre au début du texte (18-19) : parnasia laurus / parva sub ingenti matris se subicit umbra. L'image du laurier est insérée dans un catalogue botanique qui concerne les relations familiales des arbres. La première partie de la liste évoque des plantes nées de la semence déposée dans la terre ; la deuxième, fondée sur une personnification, aborde les arbres dont les rejetons puissent d'une racine de la mère. ${ }^{71}$ L'adjectif parnasia se réfère naturellement à l'amour violent d'Apollon envers Daphné dont la transfiguration est un sujet traité aussi dans les Erotica Pathémata (15). Sans parler d'un emprunt direct, la philologie ancienne met en parallèle l'image virgilienne du laurier, présente aussi dans les Bucoliques, avec le récit parthénien. ${ }^{72}$ Néanmoins, la version grecque du mythe se diffère de celle latine généralement connue grâce aux Métamorphoses d'Ovide. ${ }^{73}$ Parthénios entrelace deux narratives. Un jeune homme endosse des vêtements féminins afin de pouvoir s'approcher de Daphné, compagnonne de la chasse d'Artémis. Sa tricherie est dévoilée et punie par un Apollon

${ }^{68}$ Serv. ad Georg. I 399 : Ceyx, filius Luciferi, habuit uxorem Alcyonen. a qua cumprohibitus isset ad consulendum Apollinem de statu regni sui, naufragio periit. cuius corpus cum ad uxorem delatum fuisset, illa se praecipitavit in pelagus. postea miseratione Thetidis et Luciferi conversi sunt ambo in aves marinas, quae alcyones vocantur....

${ }^{69}$ Voir les détails chez LiGHTFOOT (n. 17) pp. 520-522.

${ }^{70}$ Servius transmet une histoire d'amour aussi à propos de lotos du vers II 84 : Lotos nympha quaedam fuit, quam cum amatam Priapus persequeretur, illa deorum miseratione in arborem versa est... 2005 , p. 71

${ }^{71}$ Voir NAPPA, CH. : Reading after Actium. Vergil's Georgics, Octavian, and Rome. Ann Arbor

${ }^{72}$ Cf. Probus, Comm. ad Ecl. III 62 : Laurus Phoebo grata, quod Daphnen ut vult Parthenius in volumine quod ei de amantibus compositum est. Chez les modernes, la ressemblance est signalée chez CADILI, sans un examen détaillé (n. 33) 182, n. 62. Servius (ad II 18), curieusement, cette fois ne parle pas d'amour : A Parnaso monte Apollini consecrato.

${ }^{73}$ Pour les détails voir LightFoot (n. 17) pp. 471-478; CAMERon (n. 39) pp. 206 et 259 ; FRANCESE (n. 20) pp. 78-79. 
jaloux, également épris de la belle. (À noter : le dieu ne figure nulle part ailleurs chez Parthénios). Après avoir annihilé son rival, Apollon poursuit l'objet de son désir sans l'obtenir; Daphné est changée en arbre ${ }^{74}$ par la clémence de Zeus. Le récit aurait une place à part dans la collection parthénienne où l'amour entre mortels et dieux ne figure qu'exceptionnellement, au contraire des réminiscences érotiques des Géorgiques qui sont dominées par des relations entre les races diverses des hommes et des dieux.

Le passage virgilien ne développe pas le mythe de Daphné, mieux, il paraît consciemment négliger toute connotation érotique. Contrairement à la future version ovidienne avec le père divin de Daphné ayant un rôle important, le poète des Géorgiques met en relief l'amour maternel par l'image des feuilles qui cachent et protègent une petite (parva) probablement en détresse. L'omission virgilienne d'un Apollon sans maîtrise de soi qui correspondrait au portrait du dieu chez Parthénios (EP XV 17-18

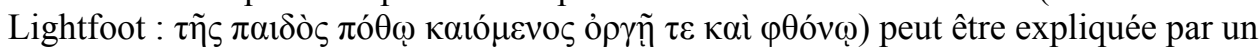
choix délibéré. Le nom d'Apollon ne se présente directement dans les Géorgiques que deux fois dans le livre final. Premièrement, il est mentionné dans le contexte poétique de l'invocation (IV 6-7) où Virgile parle de la gloire non tenuis qui est accessible par une élaboration minutieuse (in tenui labor) du sujet conjointe avec la bienveillance du dieu sollicité (auditque vocatus Apollon). Le nom divin fait son retour à la fin du livre dans le monologue d'Aristée, fils d'Apollon (IV 323 : Thymbraeus Apollon). Ce morceau est fondé sur une mise en abyme narrative remarquable. Aristée, tout comme son père Olympien, poursuit une nymphe, Eurydice. (Cette dernière, pourtant, n'a pas la chance de s'échapper à la convoitise violente par une transfiguration en arbre.) On doit noter que le portrait d'Apollon subit un changement notable dans les années de l'accès au pouvoir d'Aguste. ${ }^{75}$ Olympien d'importance mineure dans la littérature de la période pré-impériale, Apollon devient le dieu majestueux de l'ordre, de l'harmonie, de l'équilibre. Ainsi, on est tenté de dire qu'une éventuelle représentation d'un Apollon pris par une passion érotique irrésistible pourrait insulter l'empereur qui s'identifie publiquement avec le dieu.

Le personnage elliptique d'Apollon est présente aussi dans le prélude déjà mentionné du chant III qui se fonde sur la technique rhétorique d'omissio. Au lieux de raconter des histoires archiconnues (4: omnia vulgata), Virgile opte pour un mythe contemporain en proposant l'ecphrase d'un futur templum dédié à César. L'image d'un Apollon paternel qui facilite le développement des êtres en voie de s'agrandir, de s'épanouir se lit dans le vers 36 du prélude : Troiae Cynthius auctor. Cette idée latente de la croissance, de la grandeur conjointe à l'image de l'empereur par excellence augustus, peut évoquer probablement aussi le petit laurier du chant II qui est protégé par sa mère contre des menaces pas définies. Quant au futur édifice religieux préféré aux mythes grecs démodés, la recherche virgilienne met en relief avec justesse les enjeux poétiques primordiaux de ce monument imaginaire. Pour n'en citer que deux études, la lecture de Thomas met en relation la description virgilienne avec les

\footnotetext{
${ }^{74}$ Pour la métamorphose des filles en arbre en général et pour le mythe de Daphné en particulier voir Forbes IRVING, P. M. C. : Metamorphosis in Greek Myths. Oxford 1990, pp. 261-263.

${ }^{75}$ Pour un examen détaillé voir la monographie de Miller, J. F. : Apollo, Augustus, and the Poets. Cambridge 2009. Pour le mythe de Daphné ibid. pp. 344-349.
} 
travaux de reconstruction du sanctuaire de Zeus en Olympie effectués par l'ordre d'Octavien. Ainsi, le temple virtuel des Géorgiques serait le pendant contemporain latin de ce modèle archaïque grec, avec César-Octavien comme un Zeus moderne. ${ }^{76}$ L'étude de Nappa insiste d'une part sur le rôle central d'Hercule dans les mythes contestés, en suggérant l'idée virgilienne d'un portrait d'Octavien en Hercule. D'autre part il met en relief les réminiscences de la poésie de Quintus Ennius, par lesquelles le texte inviterait le lecteur à établir une nouvelle analogie entre Ennius, désormais possesseur et Virgile, nouveau candidat de l'immortalité poétique. ${ }^{77}$

Parmi les mythes rejetés dans le prologue du chant III, Virgile se réfère aussi à deux récits d'amour vus comme des histoires mille fois redites qui ont complètement perdu leur fascination littéraire (III 6-7 : cui non dictus Hylas / Hippodameque umeroque Pelops insignis eburno ?). Les trames narratives du mythe d'Hylas et de celui du couple fondateur des jeux olympiques paraissent être présentes aussi dans les Erotica Pathémata. La disparition du bien-aimé d'Hercule qui a été enlevé auprès de la source par des nymphes éprises d'amour pour lui ne figure pas dans les recueil (cependant, elle a été très probablement traitée dans fr. 32 Lightfoot). Or, le recueil transmet un récit où le motif de la mort quasiment accidentelle d'un jeune dans l'abysse de l'eau est bien présent (EP XIV). En suivant un poème d'Alexandre L'Étolien, Parthénios, proposerait une version sécularisée du rapt d'Hylas qui est une nympholepsie par excellence. Selon son récit mis dans un contexte civilisé et urbain, la reine de Milète veut séduire Antheus, otage noble de son mari, qui n'accepte pas cette invitation à un rapport illégal. (Si on veut, le récit est un amalgame du mythe de Phèdre et du thème parthénien déjà vu d'aimer son ennemi.) Pour le venger, la femme offensée prie le jeune aux mœurs excellents de descendre dans un puits sous prétention de rechercher son favori, une perdrix apprivoisée disparue. ${ }^{78}$ À peine descendu, le jeune homme est écrasé par une pierre énorme jetée sur lui par la reine. La présence elliptique du motif du rapt d'Hylas à l'arrière-plan de la narration peut être prouvée par une information à première vue sans importance. Selon la variante du mythe dans une élégie d'Alexandre L'Étolien, citée longuement par Parthénios, l'objet perdu à retrouver dans la profondeur de l'eau serait un seau d'or. ${ }^{79}$ Le motif de la cruche évoque la scène de la source du mythe d'Hylas, tant fois rédigée par les auteurs hellénistiques. L'histoire de l'amour meurtrier se finit chez Parthénios par le motif de la contrition. La reine, toujours incapable d'éteindre sa passion, se suicide ; le roi, tourmenté par la transgression malgré lui de la loi d'hospitalité, quitte le pouvoir.

Au centre du deuxième mythe érotique mis à côté par Virgile se trouve le motif de l'amour glorieux qui surmonte les obstacles par la tricherie. Selon la tradition gardée

\footnotetext{
${ }^{76}$ Voir THOMAS (n. 14) pp. 44-49.

${ }^{77}$ Voir NAPPA (n. 71) pp. 116-117, avec un excellent compte rendu sur la réfutation du modèle pindarique et callimachéen.

${ }^{78}$ À noter : la perdrix a une connotation négative dans l'éthique zoologique de l'antiquité à cause de son impulsion sexuelle particulièrement forte.

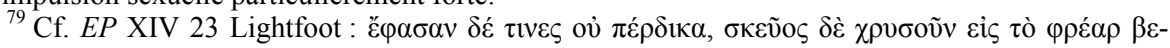



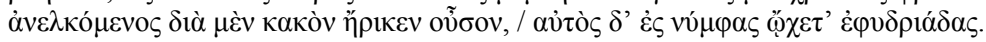


aussi par Servius, ${ }^{80}$ le mariage de Pelops et d'Hippodamie est assuré par une mise en scène macabre qui, maquille un meurtre en accident, tout comme on l'a vu ci-dessus dans l'histoire d'Antheus. Les prétendants d'Hippodamie doivent se battre à la course en char avec le roi Enomaos. Éprise du désir envers Pelops, la princesse soudoie Myrtilos, le cocher de son père en lui promettant la première nuit nuptiale. Or, après la victoire de Pelops, le jeune couple ne tient pas son serment et tue l'aurige en le précipitant dans la mer.

Une histoire tout à fait similaire se lit dans le chapitre VI du recueil parthénien qui réutilise aussi le motif de l'amour d'ennemi. La jolie Palléné, princesse de la Thrace est demandée en mariage par deux jeunes qui doivent s'engager dans un concours hippique afin de gagner la main de la fille. Tombée amoureuse d'un de ses prétendants, Palléné par l'intermédiaire de sa nourrice corrompe l'aurige du concurrent afin de faciliter la victoire de son favori par la mort de l'autre candidat. Son père, roi juste de son pays, ayant appris l'intrigue de sa fille, la condamne au bûcher. Palléné est sauvée par une intervention divine ; les flammes sont éteintes par la pluie et le mariage des amoureux peut avoir lieu. Bien que plusieurs détails narratifs et poétiques divergent dans les deux récits, ${ }^{81}$ le mythe grec de Palléné et l'évocation virgilienne d'Hippodamie semblent se fonder sur la même structure narrative. Un autre point à noter : le nom de Palléné fait son retour à la fin du livre IV des Géorgiques. Ville de la péninsule Chalcidique, Palléné est la patrie de Proteus, vieillard omniscient de la mer, ${ }^{82}$ qui joue un rôle définitif dans l'histoire d'Aristée.

La première partie du chant IV des Géorgiques développe des sujets concernant l'apiculture. En parlant des ennemis naturels des abeilles, le poète mentionne l'hirondelle par une allusion étiologique (14) : et manibus Procne pectus signata cruentis. Le passage sur l'oiseau aurait une importance narrative. Selon une lecture moderne, l'image de l'hirondelle qui nourrit ses petits $(17$ : ore ferunt dulcem nidis immitibus escam) établit un rapport entre l'instinct animal et le comportement délibéré de l'homme ; ainsi, elle prépare la description de la vie de la ruche, marquée par un vocabulaire humanisant. ${ }^{83}$ Tout de même, la scène ornithologique avec la mère qui alimente son nid s'oppose directement au personnage de la mère maltraitée et meurtrière et de l'enfant dévoré du mythe grec caché. En plus, l'image de l'hirondelle aurait un rôle aussi pour la structure du livre IV, car elle forme un cadre narratif avec le grand récit final des Géorgiques : en représentant Orphée en deuil, Virgile évoque le chant funèbre du rossignol (511-515: maerens philomela sub umbra). L'histoire de Procné et de Philomèle appartient aux couches les plus anciennes de la mythologie

${ }^{80}$ Cf. Serv. ad Georg. III 7 : ... postea cum Pelopem amasset Hippodamia, corrupit Myrtilum, aurigam patris, primi coitus pactio, qui factis cereis axibus cum victore Pelope a puella promissum posceret praemium, ab eius mari praecipitatus in mare est, cui nomen imposuit.

${ }^{81}$ Voir par exemple l'allusion au modèle pindarique chez Virgile (ainsi, le portrait de Pelops umero insignis eburno évoquerait sa relation amoureuse avec Poséidon qui est racontée par la Première Olympique), ce qui s'oppose aux réminiscences d'Hérodote chez Parthénios. Pour le récit sur Palléné en général voir LIGHTFOOT (n. 17) pp. 403-407.

${ }^{82}$ Cf. Georg. IV 390-391 : hic nun Emathiae portus patriamque revisit / Pallenen...

${ }^{83}$ Cf. NAPPA (n. 71) p. 163. 
grecque, ${ }^{84}$ par conséquence, elle ne fait pas partie de la collection parthénienne. Néanmoins, le motif de l'infanticide conjoint avec celui de l'anthropophagie (qui est généralement le châtiment d'un autre crime familial, notamment de l'inceste), est bien présent dans les Erotica Pathémata.

Ainsi, une des trois métamorphoses parthéniennes, l'aition du chapitre 13 présente le mythe d'origine de l'oiseau $\chi \alpha \lambda$ kíc. Une certaine Harpalyké est forcée aux relations incestueuses par son père qui annule le mariage de sa fille avec un brave jeune homme. (Par son nom parlant, Alastor peut être vu comme l'agent de la justice divine.) Les noces échouées, la fille malheureuse tire vengeance par l'assassinat de son petit frère qui, coupé en morceaux, est mis à la table de leur père. Fait accompli, Harpalyké prie les dieux de mettre fin à sa souffrance qui la transfigurent en oiseau. Concernant les éventuelles relations entre Parthénios et Virgile, on devrait ajouter

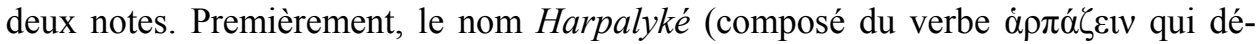
signe une activité prédatrice et du nom du loup) fait son retour chez Virgile dans un contexte tout à fait différent, notamment dans la scène lybienne de son épopée. Chez Parthénios, Harpalyké est la victime innocente d'un acte brutal et sauvage qui est l'inceste. Puis, déformée par son entourage, elle réalise un acte pas moins horrible. Par contre, dans la métaphore de l'épiphanie de Venus au chant I de l'Énéide, Harpalyké est représentée en chasseuse de Thrace, en venatrix qui tue des bêtes ${ }^{85}$ par un geste naturel. (Ce caractère meurtrier d'Harpalyké paraît être évoqué aussi par l'image d'une autre fille sauvage : dans le catalogue de l'aristée de Camille on retrouve un victime nommé Harpalycus, Én. XI 676). Deuxièmement, la transformation en oiseau est un moyen narratif typique aussi chez Virgile pour conclure un récit d'inceste. ${ }^{86}$ Ainsi, le vers 403 du chant I (nequiquam seros exercet noctua cantus) fait entendre la voix inquiétante de la chouette qui rappelle le mythe de Nyctimène, elle aussi objet de la pulsion incestueuse de son père. ${ }^{87}$

L'histoire d'Orphée et d'Eurydice, le seule mythe érotique traité en profondeur dans les Géorgiques, ne peut pas être analysée ici en détail. On doit se borner à souligner le plus important : bien que le poète n'accuse pas ouvertement Aristée d'une tentative du viol, le fils d'Apollon est responsable de la mort à première vue accidentelle d'Eurydice, et, indirectement, de celle d'Orphée. La question de la proportionnalité de la peine au délit (la mort irrévocable des hommes contre la disparition, ensuite la résurrection d'un essaim d'abeilles) reste un problème très disputé.

${ }^{84}$ Cf. Serv. ad Georg. IV 14 : nomen posuit pro nomine; nam Philomela in hirundinem versa est: pro qua Procnen vel quasi sororem posuit, vel quasi eam, quae fuerat illius sceleris causa; nam ipsa Tereum miserat ad adducendam sororem.

${ }^{85}$ Cf. Én. I 316-317 : Cui mater media sese tulit obvia silva / virginis os habitumque gerens, et virginis arma / Spartanae, vel qualis equos Threissa fatigat / Harpalyce, volucremque fuga praevertitur Hebrum.

${ }^{86}$ Pour la métamorphose des filles en oiseaux en guise d'apothéose voir ForBeS IRVING (n. 74) p. 115 sqq. Pour les sources du mythe parthénien d'Harpalyké voir LightFOOT (n. 17) pp. 446-454 ; CAMERON (n. 39) pp. 108-109 ; FRANCESE (n. 20) p. 137.

${ }^{87}$ Cf. Serv. ad Georg. IV 403 : Nyctimone postquam cum patre concubuit et agnovit facinus esse, in silvis se abdidit et lucem refugit, ubi deorum voluntate conversa est in avem, quae pro tanto facinore omnibus avibus est admirationi. 
Parmi les dernières allusions mythologiques du chant IV, on doit remarquer le morceau sur la sympathie de la nature qui partage le deuil d'Orphée. Eurydice, disparue, est pleurée par le chœur des nymphes, par des montagnes et des rochers. Afin d'exprimer la tristesse cosmique, un petit catalogue géographique ${ }^{88}$ donne une vue panoramique fondée entre autres sur trois histoires d'amour. Le mythe de Rhodope et de son mari gagnera son ampleur dans le futur récit d'Ovide, mais à cause de la personnification rhétorique (461 : flerunt Rhodopeiae arces) on aurait raison de supposer que Virgile aussi le connaît déjà. Les conjoints fous du bonheur se nomment Jupiter et Junon, leur transformation en montagnes est la punition de leur orgueil. ${ }^{89}$ Bien que Haîmos et Rhodope ne soient mentionnés nulle part dans le recueil grec, on ne peut pas exclure une version parthénienne de leur histoire. ${ }^{90}$

Leur mythe d'amour est suivi par une allusion à celui de Rhésus (462: Rhesi Mavortia tellus). Par la connotation militaire de l'adjectif Mavortia, la phrase paraît évoquer plutôt l'assassinat ignoble de Rhésus à Troie, épisode bien connu de la tradition homérique. Néanmoins, on est tenté de soupçonner aussi la présence du motif du bonheur conjugal d'autrefois de Rhésus et de sa femme; cette émotion rendrait capable le pays du Rhésus d'avoir la compassion pour Orphée et Eurydice. Le mariage heureux de Rhésus et d'Arganthoné est le dernier mythe traité chez Parthénios $(E P X X X V I) .{ }^{91}$ Le couple formé du chasseur et de la chasseuse se retire de la civilisation pour vivre caché dans la nature. Leur félicité ne se termine que par la mort du mari qui est suivie du décès volontaire de son épouse affligée par la douleur qui se suicide en refusant de s'alimenter. Leur mythe, d'ailleurs, formerait un cadre narratif avec le mariage mouvementé mais solide de Lyrcos et d'Hilébie au chapitre I du recueil, en concluant la série des passions amoureuses. (Le troisième mythe d'amour évoqué par Virgile dans la scène du deuil pour Eurydice, celui du rapt d'Orythie par Borée [463 : Actias Orithyia] n'aurait pas un pendant dans le recueil parthénien et remonterait à Platon [Phèdre 229b-c]).

Sans pouvoir parler avec certitude d'une influence parthénienne directe ou exclusive sur l'auteur des Géorgiques jusqu'ici on a énuméré des allusions virgiliennes qui se mettraient facilement en parallèle avec certains types de récits des Erotica Pathémata. En revanche, certaines allusions aux mythes érotiques qui forment chez Virgile des groupes thématiques et qui semblent avoir un impact non négligeable sur son concept d'amour, ne se trouvent pas chez Parthénios.

Comme il a déjà été souligné, à l'exception de l'histoire de Daphné, dans le recueil grec ne se lisent pas des récits qui mettent en relation intime des dieux avec des mortel(le)s. Dans leur grande majorité, les protagonistes de Parthénios sont des hommes et des femmes terrestres. À cause de cette absence des amours métis, dans les Erotica Pathémata on chercherait en vaine le personnage de la déesse jalouse qui punirait les

\footnotetext{
${ }^{88}$ Cf. Georg. IV 461-462 : ... flerunt Rhodopeiae arces / altaque Pangaea et Rhesi Mavortia tellus / atque Getae atque Hebrus et Actias Orithyia.

${ }^{89}$ Servius passe sous silence l'amour de Rhésus, cf. ad Georg. IV 462 : est prolepsis ex persona poetae: quo enim tempore Orpheus fuit, Rhesus necdum regnabat in Thracia.

${ }^{90}$ Voir FRANCESE (n. 20) p. 137 qui suppose un amour entre frère et sœur.

${ }^{91}$ Pour les détails voir LiGHTFOOT (n. 17) pp. 552-558.
} 
femmes terrestres, victimes du désir du roi Olympien infidèle. En revanche, les allusions virgiliennes sont largement dominées par le motif du mélange érotique des dieux et des femmes mortelles. À noter : le seul homme envieux de l'amour d'une déesse serait Ixion, châtié pour son attentat à la pudeur de Junon (III 38 : tortosque Ixionis anguis). ${ }^{92}$ Chez Virgile, on ne trouve qu'une seule histoire d'amour entièrement humaine et sécularisée, celle d'Héro et de Léandre qui est également un mythe étiologique. ${ }^{93}$ En ce qui concerne le motif récurrent de la femme terrestre séduite malgré elle par un dieu et torturée par l'épouse divine jalouse, on pourrait citer de nombreux exemples. Ainsi, deux des étoiles qui forment la constellation de la Grande Ourse sont identifiées par Servius avec Héliké et Cynosura. Selon une de ses explications alternatives, les filles choisies pour le lit de Zeus ont été transformées en astres par Junon (I 264 : Arctos Oceani metuentis aequore tingi). ${ }^{94}$ Un autre exemple s'impose par le mythe de Philyra, mère du centaure Chiron qui est changée en jument (ou bien en tilleuil) par son amant Cronos afin qu'ils puissent déjouer la surveillance de la conjointe divine. ${ }^{95}$

L'histoire d'Io, changée en génisse et tourmentée sans arrêt par sa rivale immortelle, est particulièrement importante aussi d'un point de vue esthétique. Le mythe est évoqué chez Virgile à propos du taon, ennemi des vaches (III 152-153 : hoc quondam monstro horribilis exercuit iras / Inachiae Iuno pestem meditata iunencae). ${ }^{96} \mathrm{La}$ toute première phrase de la collection parthénienne mentionne également Io et sa disparition, tout en proposant une variante étonnante selon laquelle la princesse d'Argos a été enlevée par des pirates. ${ }^{97}$ Or, le récit grec met de côté immédiatement les souffrances d'Io pour continuer avec les passions beaucoup moins tragique de Lyrcos, héros peu connu qui, sous l'ordre du roi Inachos, doit partir à la recherche de la fille royale. Les aventures de Lyrcos qui sont majoritairement des expériences érotiques s'achèvent sur un mariage qui est heureux et stable (malgré l'infidélité involontaire de Lyrcos). ${ }^{98}$

\footnotetext{
${ }^{92}$ Serv. ad Georg. III 38-39 : tortosque ixionis: a quibus religatus ad rotam est, postquam inlicitos Iunonis petivit amplexus.

${ }_{93}^{93}$ Georg. III 258-263 : quid iunenis, magnum cui uersat in ossibus ignem / durus amor? nempe abruptis turbata procellis / nocte natat caeca serus freta, quem super ingens / porta tonat caeli, et scopulis inlisa reclamant / aequora; nec miseri possunt reuocare parentes, / nec moritura super crudeli funere uirgo.

${ }^{94}$ Serv. ad. Georg. I 246 : nam hae duae paelices Iunonis fuisse dicuntur: quas postquam Iuppiter in siderum rettulit numerum, Iuno rogavit Tethyn, suam nutricem, ne umquam eas pateretur occidere. unde nunc 'metuentes' dixit, scilicet nutricem Iunonis. sunt autem hae Helice et Cynosura. Les variantes proposées concernent également de mythes érotiques. Héliké est une nymphe métamorphosée en ourse par Junon jalouse ; Cynosura, compagnonne de chasse d'Artemis qui perd sa virginité, est transformée également en ourse par la déesse irritée, leur catastérisme postérieur se réalise par la clémence des dieux.

${ }^{95}$ Serv. ad Georg. III 92-94 : talis et ipse iubam ceruice effundit equina / coniugis aduentu pernix Saturnus, et altum / Pelion hinnitu fugiens impleuit acuto, cf. Serv. ad locum : dum cum amata Philyra Saturnus coiret, Ops eius uxor advenit. cuius praesentiam veritus, se in equum convertit, qualem potuit numen imitari. exinde natus est Chiron, dimidia parte homo, dimidia equus....quidam Philyram in florem conversam dicunt vel in arborem, unde liber philyrinus dicitur... Pour le mythe de Philyra voir NAPPA (n. 71) p. 127.

${ }^{96}$ Serv. ad Georg. III 153 : pestem meditata ivuencae: Ionem dicit, Inachi filiam, quam in bovem conversam (Iuno) oestro percussisse dicitur. illa se in mare praecipitavit, (quod) Ionium vocaverunt..

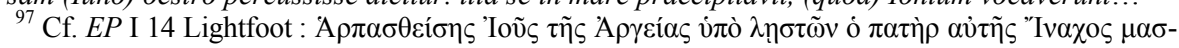

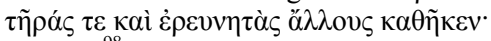

${ }^{98}$ Pour le récit en général voir LightFoOT (n. 17) pp. 371-379.
} 
Cette nouvelle version du mythe d'Io, placée à l'entrée du recueil et caractérisée par un changement $\mathrm{du}$ focus très remarquable, aurait une valeur programmatique. L'intention poétique de Parthénios n'est de rabâcher des histoires connues par tous (comme la séduction et la métamorphose d'Io), mais de corriger les mythes traditionnels en examinant leurs faces cachées pleines de secrets, ${ }^{99}$ ou bien de mettre en relief les personnages jusqu'ici ignorés de la mythologie. La question rhétorique au prologue du chant III des Géorgiques - cui non dictus Hylas puer ? - semble parfaitement concorder avec ce programme littéraire suggéré de Parthénios.

Pour continuer de passer en revue les motifs érotiques primordiaux du poème latin, la mort accidentelle d'un beau jeune aimé par un dieu est également une trame typique des mythes cachés des Géorgiques qui ne se présente pas dans les Erotica Pathémata. Le tout premier mythe d'amour de l'œuvre dans l'invocation des dieux champêtres fait voir Silvaine une branche de cyprès à la main (I 20) : et tenaram ab radice ferens Silvane, cupressum. Selon le commentaire de Servius, le vers se réfère au jeune Cyparissos inconsolable parce que son beau cerf a été mortellement blessé par son amant, dieu des forêts. ${ }^{100}$ Mort du chagrin, le jeune, est transfiguré en cyprès. Bien que le nom de Cyparissos ne figure pas dans le recueil grec qui par sa tendance de rationaliser évite régulièrement le motif de la métamorphose, ${ }^{101}$ son histoire rappelle les scènes de la chasse en majorité tragiques des Erotica Pathémata (voir EP X, XV, XVI, XXXII, XXXIII, XXXVI). À noter : le motif d'une bête hors pair dont l'extinction est le point de départ des événements graves peut être considéré comme un modèle possible de l'épisode du cervus forma praestanti de Silvie dont la mort devient casus belli pour les Latins au chant VII de l'Énéide (482 sqq.).

Insérée dans le contexte d'apiculture, l'image virgilienne du narcisse qui pleure (IV 160 : narcissi lacrimam) rappelle également un mythe d'amour tragique. ${ }^{102}$ Dans sa version originale, pré-ovidienne a été probablement fondé sur une relation incestueuse entre frère et sœur. L'histoire ne se trouve pas dans les Erotica Pathémata, pourtant, selon le témoignage d'une découverte papyrologique (P.Oxy 4711), ${ }^{103}$ elle a été élaborée chez Parthénios. Toujours dans le contexte de l'apiculture, la description du pré fleuri visité par des abeilles est complétée par la jacinthe sombre (IV 183 : ferrugineos hyacinthos), en faisant allusion à son mythe d'origine connu. Meurtrier malgré lui, Apollon, tue son bien-aimé pendant un lancer de disque; la couleur

${ }^{99}$ Pour un autre exemple voir l'histoire d'Assaon et de sa fille Niobé (EP XXXIII). Parce que Niobé, veuve d'un homme vertueux, ne cède aux pulsions incestueuses de son père, Assaon séquestre et brûle vivants ses petits-enfants. Affligée par la douleur maternelle, Niobé se jette à la mer.

${ }^{100}$ Serv. ad Georg. I 20 : Silvanus deus est silvarum. hic amavit puerum Cyparissum nomine, qui habebat mansuetissimam cervam. hanc cum Silvanus nescius occidisset, puer est extinctus dolore: quem amator deus in cupressum arborem nominis eius vertit, quam pro solacio portare dicitur... Dans la version ovidienne l'amant divin de Cyparissos est Apollon.

101 Voir LightFoOT (n. 17) p. 230.

${ }^{102}$ Serv. ad Georg. IV 160 : Allusit ad fabulam, quia de puero est conversus in florem.

${ }^{103}$ Cf. P. Oxy 4711, fr. 1, 8. Voir aussi MAgNELLi (n. 28) pp. 9-12. Contre l'attribution parthénienne du fragment sur Narcisse voir BERNSDORFF, H. : P. Oxy. 4711 and the Poetry of Parthenius. JHS 127 (2007) pp. 1-18. 
rougeâtre de la fleur garde le sang coulé du corps du jeune. ${ }^{104}$ Le mythe d'Hyacinthe, absent dans les Erotica Pathémata, fait partie d'un catalogue des métamorphoses homoérotiques attribué à Parthénios qui se lit sur un fragment papyrologique (P.Oxy 2723) récemment trouvé.

En ce qui concerne finalement la dimension éthique des textes examinés, un enseignement moral régulier n'est pas visible chez Parthénios. Bien que l'éditio princeps des Erotica Pathémata publiée en 1531 considérait l'œuvre comme un recueil pédagogique qui proposerait à la jeunesse chrétienne des exemples à ne pas suivre, ${ }^{105}$ Parthénios garde une attitude réservée et il ne s'exprime pas sur la valeur éthique des actions de ses protagonistes (sauf son reproche étonnant à propos de l'immoralité d'Ulysse aux chapitres II-III). Tout comme la mise en forme poétique, Parthénios laisse l'élaboration morale de ses histoires à son lecteur. Malgré cette position moralement neutre, sa compilation formée des récits qui sont souvent marqués par la lutte entre pudeur et désir paraît avoir un petit penchant à une vue plutôt pessimiste sur les mœurs humains. ${ }^{106}$ Les crimes motivés par la folie amoureuse dépassent plusieurs fois la volonté libre de l'individu et ils peuvent avoir de graves conséquences aussi pour des communautés entières. ${ }^{107}$ Les péchés d'origine érotique sollicitent des actes encore plus horribles (voir l'association étroite de l'inceste avec l'homicide, EP XIII et XXXIII) et dans la plupart des cas ils restent sans châtiment. Les innocents meurent, les criminels vivent heureux : l'intervention d'une justice terrestre ou divine ne fait apparition que très rarement $\left(E P X V\right.$ et XXVII). ${ }^{108}$ Tout de même, on doit concéder : à côté des familles incestueuses (EP V, XI, XIII, XVII, XXXIII), des tyrans cruels (VII), dans le recueil font apparition aussi des pères qui aiment leur filles sans les désirer, des barbares nobles (VIII), on trouve des femmes mortelles et des nymphes qui aiment leurs maris même adultères (I, IV), des mourants qui pardonnent à leurs assassins (XXIV). Même l'inceste peut avoir une certaine valeur, comme on l'a vu dans l'histoire de Polymèle : déshonorée par Ulysse et condamné à mort par son père, elle est sauvée par un mariage avec son frère $(E P \mathrm{II}){ }^{109}$

En revanche, dans les mythes des Géorgiques on constate une mise en profondeur éthique tout à fait inconnue chez Parthénios. Les allusions virgiliennes qui sont beaucoup plus courtes que les chapitres déjà abrégés du recueil grec semblent dépasser le rôle d'ornement et gagner une dimension théologique par la forte présence des

\footnotetext{
${ }^{104}$ Serv. ad Georg. IV 183 : Hoc poetice masculino, referens se ad puerum qui in hunc florem dicitur esse conversus.

${ }^{105}$ Voir LightFoot (n. 17) p. 221.

106 À noter : l'auteur de l'épigramme contre Parthénios ( $A P$ VII $377=$ T 2 Lightfoot) semble rejeter l'œuvre parthénienne aussi par des raisons éthiques, pour les détails voir KLOOSTER (n. 40) p. 319.

${ }^{107}$ Pour les enjeux sociaux de l'amour illégitime voir l'histoire de Périandre : homme doux et raisonnable, à la suite de sa relation incestueuse provoquée par sa mère il devient un tyran sanguinaire ( $E P$ XVII). Un autre récit s'oppose nettement à cet amour aux conséquences désastrueuses pour la polis. La relation homoérotique d'Hipparinus et d'Antileon, menacée par le tyran jaloux de leur ville, s'achève sur le tyrannicide et l'acte de tyrannicide et l'exécution des jeunes amants ; toutefois, la mort du couple rend la liberté à leur communauté ( $E P$ VII).

${ }_{108}$ Voir aussi KLOOSTER (n. 40) p. 314, n. 19.

${ }^{109}$ Pour le ton presque comique du récit voir VoIsIN (n. 44) p. 50.
} 
figures divines et des métamorphoses. Une autre spécialité de la composition virgilienne est l'élargissement du thème de l'amour vers la sphère des animaux sans raison dans le livre III. Ainsi, les allusions érotiques s'intègrent dans un contexte philosophique universel qui concerne désormais le pathos commun de tous les êtres vivants : amor omnibus idem (III 244). Les mythes d'origine du poème contribuent à la vision virgilienne du monde, en proposant une certaine cosmogonie érotique. Cet objectif narratif semble être confirmé par la scène où Clymené, mère d'Aristée chante aux nymphes de la mer les amours des dieux à partir d'origine du monde. ${ }^{110}$ Similairement aux récits de Parthénios, les mythes des Géorgiques (on concède, moins excentriques ou horrifiants) sont presque toujours tragiques, liés à la notion de la cruauté, de la perfidie. Chez Virgile, tout comme dans les Erotica Pathémata, il n'y a pas d'amour heureux.

L'importance des allusions mythologiques plus ou moins elliptiques sera mise en évidence dans livre IV, d'une part par le mythe d'Orphée, d'autre part par le motif des abeilles. Selon Virgile, les insectes pures qui travaillent avec beaucoup d'application sont les seuls êtres qui ne connaissent pas l'amour et qui ne sont pas soumis aux forces dévastatrices des passions. ${ }^{111} \mathrm{C}$ 'est un fait remarquable, vu que la représentation de la vie des abeilles est généralement considérée comme le modèle d'une société humaine idéale. On dirait que la séquence des mythes érotiques paraît mettre un bémol dans une œuvre qui en accord avec les exigences d'Auguste (mentionné cinq fois dans les Géorgiques et associé toujours avec Jupiter ${ }^{112}$ ) devrait exhorter au travail, au recommencement, à la réalisation d'une nouvelle société. Il semble que Virgile par ses allusions mythologiques veut faire réfléchir ses lecteurs sur la condition humaine, sur la volonté libre et la force inéluctable de la passion.

Évidemment, on pourrait élargir l'analyse, car cette notion d'amour semble être identique avec celui des Bucoliques et de l'Énéide, marqués également par les adjectifs durus, crudelis, improbus, indignus, saevus. Pour boucler notre examen on doit se contenter de dire que les mythes d'amour cachés des Géorgiques semblent faire partie intégrante de la fortune latine des Erotica Pathémata. Selon le témoignage des corrélations des mythes d'amour évoqués par les deux auteur, l'esprit et l'imagination du poète vertueux néanmoins impatiens libidinis des Géorgiques a été probablement alimenté non seulement par la Sirène désormais silencieuse mais aussi par les narrations érotiques de Parthénios.

Elvira Pataki

Département de Langues et Littératures Anciennes

Université Catholique Pázmány Péter

Hongrie

${ }^{110}$ Cf. Georg. IV 344-347 : Inter quas curam Clymene narrabat inanem / Vulcani Martisque dolos et dulcia furta / aque Chao densos divum numerabat amores. Voir OTIS (n. 30) p. 138.

${ }^{111}$ Cf. Georg. IV 197-201: Illum adeo placuisse apibus mirabere morem, / quod neque concubitu indulgent nec corpora segnes / in Venerem solvunt aut fetus nixibus edunt: / verum ipsae e foliis natos, e suavibus herbis / ore legunt...

${ }^{112}$ Cf. Georg. I 24-42, 498-514, II 170-172, III 16-48, IV 559-562, voir aussi ThOMAS (n. 14) p. 44. 